\title{
Research Paper \\ Prevalence, Clinical Manifestation and Mortality Rate in COVID-19 Patients With Underlying Diseases
} \author{
Khorrami ${ }^{5}$, Emran Esmaeilzadeh ${ }^{1}$ [C] \\ 1. Department of Genetics and New Technologies, School of Medicine, AJA University of Medical Science, Tehran, Iran. \\ 2. Department of Biochemistry and Genetics, School of Medicine, Arak University of Medical Sciences, Arak, Iran. \\ 3. Medical Genetics Lab, Beski Hospital, Gonbad-e Kavus, Golestan, Iran. \\ 4. Department of Medical Genetics, Faculty of Medical Sciences, Tarbiat Modares University, Tehran, Iran. \\ 5. Department of Infectious Diseases, School of Medicine, Shiraz University of Medical Sciences, Shiraz, Iran.
}

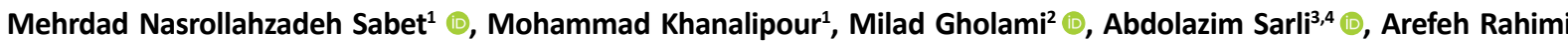

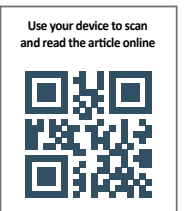

Citation: Nasrollahzadeh Sabet M, Khanalipour M, Gholami M, Sarli A, Rahimi Khorrami A, Esmaeilzadeh E. [Prevalence, Clinical Manifestation and Mortality Rate in COVID-19 Patients With Underlying Diseases (Persian)]. Journal of Arak University of Medical Sciences (JAMS). 2020; 23(5):740-749. https://doi.org/10.32598/JAMS.23.COV.5797.1

doi) https://doi.org/10.32598/JAMS.23.COV.5797.1

Key words:

Coronavirus Disease

2019, Diabetes,

Hypertension, Car-

diovascular disease, Mortality

\section{A B S T R A C T}

Background and Aim The new Coronavirus Disease 2019 (COVID-19) outbreak initiated from China in late December 2019, has posed a great global health threat. Since patients with high blood pressure, diabetes, and cardiovascular diseases are at high risk of COVID-19, this study aims to investigate the prevalence, severity, clinical manifestations, and mortality rate in COVID-19 patients with underlying diseases compared to those without underlying diseases.

Methods \& Materials This study was performed on 1408 patients with COVID-19 admitted to Golestan, Hajar, Khanevadeh and Besat hospitals in Tehran, Iran. Required data including disease prevalence, severity, clinical manifestations and mortality rate in patients with and without underlying diseases were collected and then analyzed using statistical tests in SPSS software, considering a significance level of $P<0.05$. Ethical Considerations This study was approved by the AJA University of Medical Sciences (Code: IR.AJAUMS.REC.1399.091). Ethical principles have been observed in accordance with the guidelines of the National Ethics Committee and the COPE regulations.

Results Patients were 912 men (64.8\%) and 496 women (35.2\%) aged 18-98 years (Mean age $=57.8$ years). Results reported that $27 \%$ of patients were intubated and $21.9 \%$ were died. Blood oxygen level in $53.7 \%$ of patients was less than $93 \mathrm{mg} / \mathrm{dL} ; 36.1 \%$ had a history of hypertension, $24.1 \%$ had a history of diabetes, and $21.7 \%$ had a history of cardiovascular disease.

Conclusion The severity, clinical manifestations, and mortality rate of COVID-19 patients with cardiovascular disease, diabetes and hypertension are significantly different compared to those without underlying diseases.

\section{Extended Abstract \\ tiated from Wuhan, China, has posed a great global health

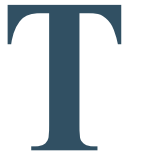 \\ 1. Introduction \\ he new coronavirus disease 2019 (Cov- id-19) outbreak in late December 2019 ini- threat. According to studies, most people with Covid-19 have underlying diseases such as high blood pressure, car- diovascular diseases and diabetes, and the mortality rate in these people is higher than in other patients [3-5]. The aim of this study is to evaluate the prevalence, severity and mor-}

\section{* Corresponding Author:}

Emran Esmaeilzadeh, PhD.

Address: Department of Genetics and New Technologies, School of Medicine, Aja University of Medical Science, Tehran, Iran.

Tel: +98 (21) 88337919

E-mail: esmaeilzadeh@ajaums.ac.ir 
Table 1. Demographic characteristics of the participants

\begin{tabular}{|c|c|c|}
\hline Characteristics & & No. (\%) \\
\hline \multirow{3}{*}{ Gender } & Male & $912(68.4)$ \\
\hline & & \\
\hline & Female & $496(35.2)$ \\
\hline \multirow{7}{*}{ Age (year) } & $<20$ & $16(1.1)$ \\
\hline & $35-21$ & $168(11.9)$ \\
\hline & $50-36$ & $324(23)$ \\
\hline & & \\
\hline & $65-51$ & 364 (25.9) \\
\hline & $80-66$ & $372(26.4)$ \\
\hline & $>80$ & $164(11.6)$ \\
\hline \multirow{3}{*}{ Smoking } & Yes & $552(60.8)$ \\
\hline & & \\
\hline & No & $856(39.2)$ \\
\hline \multirow{2}{*}{ Intubation } & Yes & $380(27)$ \\
\hline & No & $1028(73)$ \\
\hline \multirow{3}{*}{ Final status } & Recovered & $1100(78.1)$ \\
\hline & & \\
\hline & Died & $308(21.9)$ \\
\hline \multirow{3}{*}{ Blood oxygen saturation level (mg/dl) } & $<93$ & 756 (53.7) \\
\hline & & \\
\hline & $\geq 93$ & $652(46.3)$ \\
\hline
\end{tabular}

tality rate of Covid-19 in patients with underlying diseases in Iran compared to other patients.

\section{Materials and Methods}

This study was performed on 1408 patients with Covid-19 who were admitted to Hajar, Golestan, Khanevadeh and Besat hospitals in Tehran, Iran from March to June 2020. Prevalence, severity and mortality were calculated in four study groups:with a history of diabetes, with a history of cardiovascular disease, with a history of hypertension, and with no history of underlying disease. The data were analyzed in SPSS V. 26 by using descriptive statistics, paired t-test, independent t-test, and chi-square test considering a significance level of 0.5 .

\section{Results}

Of 1408 patients, 912 were male (64.8\%) and $496(35.2 \%)$ were female, with a gender ratio of $1 / 8$ and a mean age of 57.8 years (ranged from 18-98 years). Based on the results, intubation was performed for $27 \%$ of patients and $21.9 \%$ died. In terms of blood oxygen level, patients were divided into two groups, $53.7 \%$ had oxygen level $<93 \mathrm{mg} / \mathrm{dl}$ and $46.3 \%$ had oxygen level $>93 \mathrm{mg} / \mathrm{dl}$ (Table 1 ). The results of examining the clinical manifestations of patients showed that the most common symptoms were fever, cough and shortness of breath. Moreover, the most common underlying diseases were hypertension, diabetes and cardiovascular diseases. The mortality rate of patients in different groups is presented in Table 2.

\section{Discussion and Conclusion}

The purpose of this study was to investigate the prevalence, severity and mortality rate of Covid-19 among patients with underlying disorders compared to controls. The results showed that patients with diabetes, high blood pressure and cardiovascular disease had a higher risk of developing the Covid-19 than others in the community, as reported in other studies. High blood pressure is often associated with other risk factors such as cardiovascular diseases and diabetes, which in turn increases the risk of developing Covid-19 [7]. Diabetes can be also considered 
Table 2. Mortality rate of Covid-19 patients in different groups

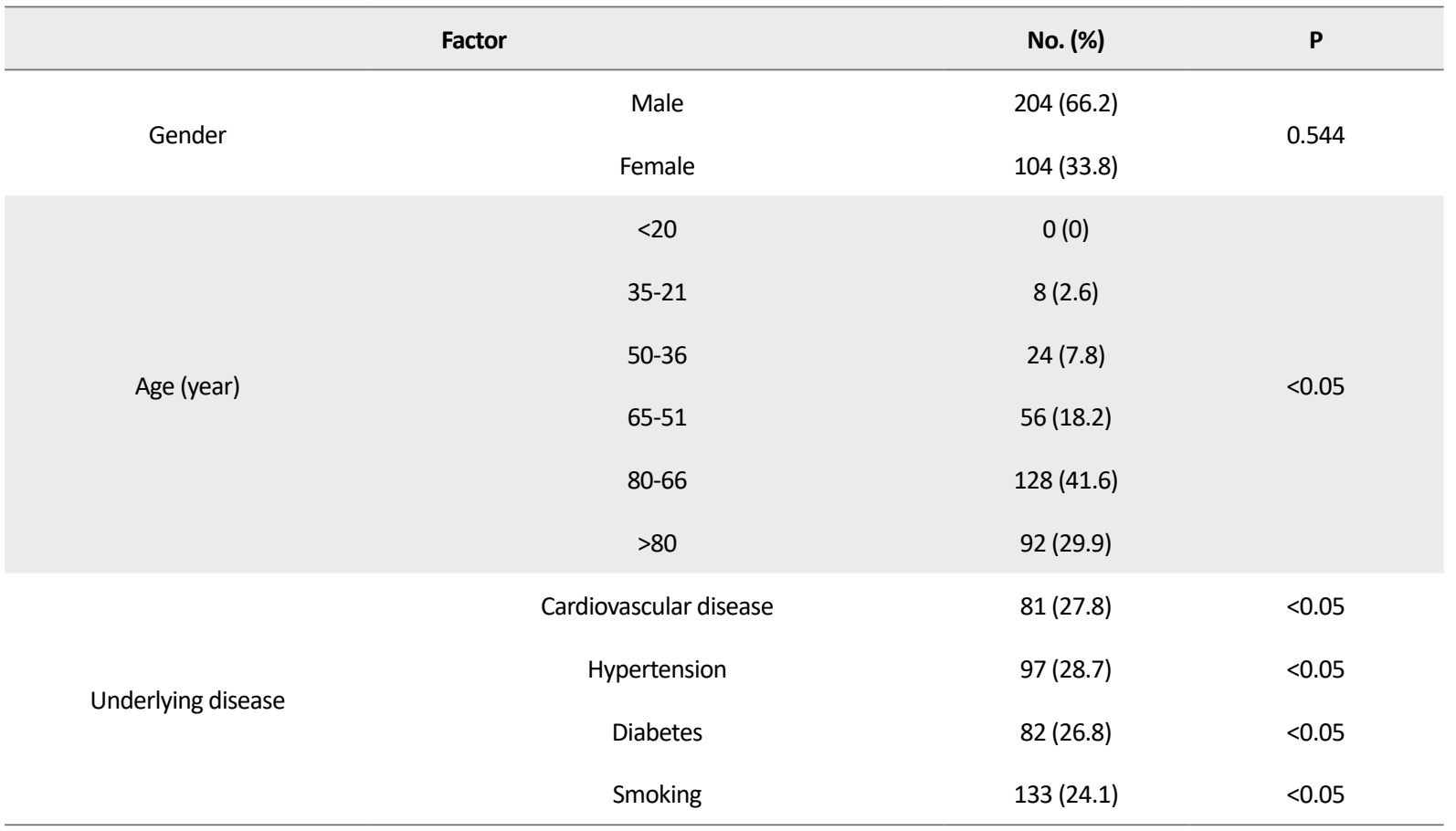

a risk factor for the deterioration of Covid-19 [4]. Previous studies have also shown that diabetes and cardiovascular disease increase the risk of SARS and MERS, which, like the Covid-19, are an acute respiratory syndrome. They increase the probability of death from SARS by $11 \%$ and $8 \%$, respectively. More than $50 \%$ of people with MERS had diabetes and hypertension and more than $30 \%$ of them had cardiovascular diseases [8]. According to the results, diabetes, high blood pressure and cardiovascular disease increased the mortality rate of Covid-19 patients. The results of this study also showed that the mortality rate in smokers with Covid-19 was higher than in controls. Therefore, it is recommended that Covid-19 patients with underlying diseases be more careful about following health safety protocols, and when a vaccine for this disease became available, people with underlying diseases should be given priority.

\section{Ethical Considerations}

\section{Compliance with ethical guidelines}

This study was approved by the Research Ethics Committee of AJA University of Medical Sciences (Code: IR.AJUMS.REC.1399.091).

\section{Funding}

This study was supported by the AJA University of Medical Science (No. 97001203).

\section{Authors' contributions}

Conceptualization, methodology, and data analysis: Emran Esmaeilzadeh and Mehrdad Nasrollahzadeh Sabet; Investigation, resources, initial draft preparation, editing \& review: Mohammad Khanalipour, Milad Gholami, Abdolazim Sarli, Arefeh Rahimikhorrami.

\section{Conflicts of interest}

The authors declare no conflict of interest.

\section{Acknowledgements}

The authors would like to thank School of Medicine, AJA University of Medical Sciences for their financial support. 


\title{
بررسى تظاهرات بالينى و ميزان مركتومير در بيماران مبتلا به كوويد 19 داراى بيمارى زمينهاى
}

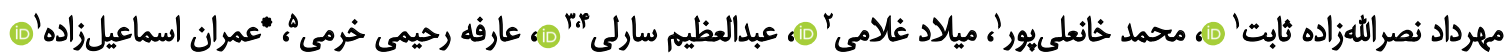

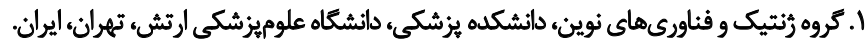

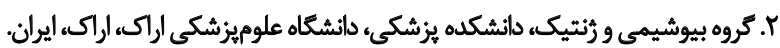

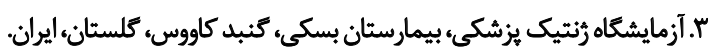

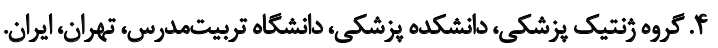

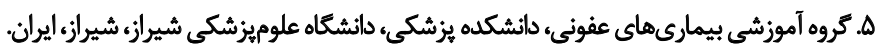

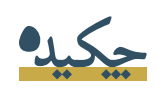

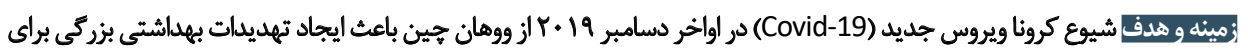

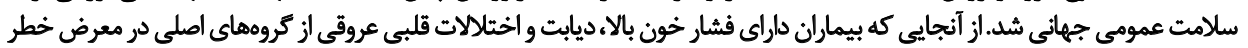

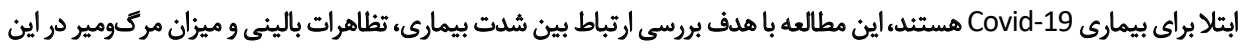

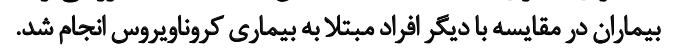

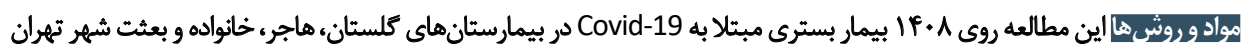

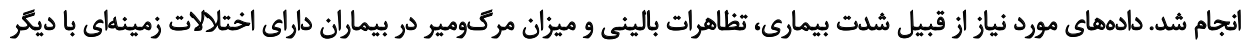

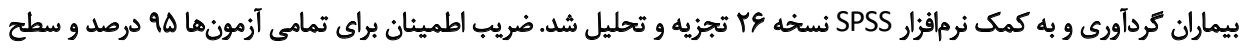

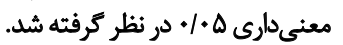

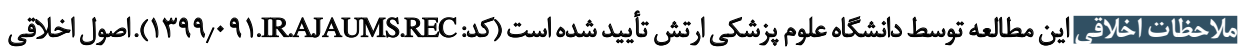

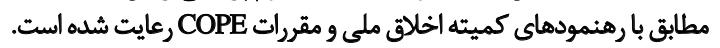

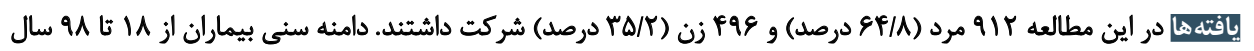

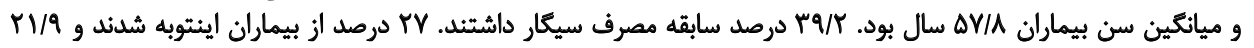

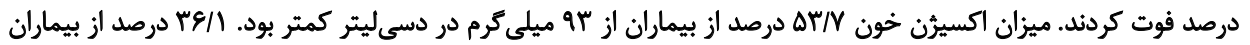

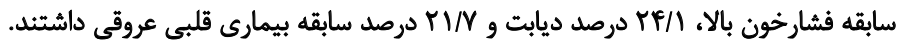

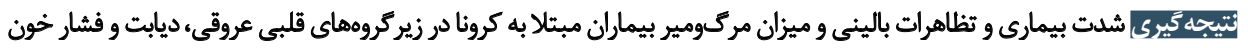

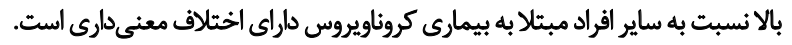

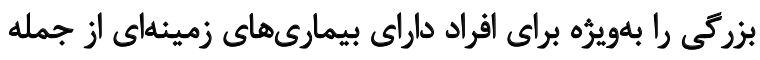

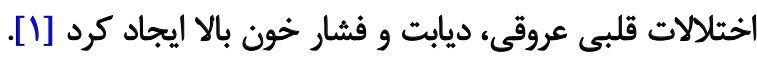

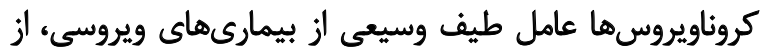

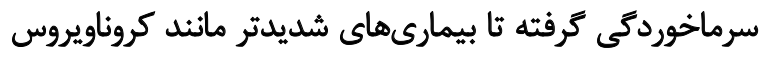

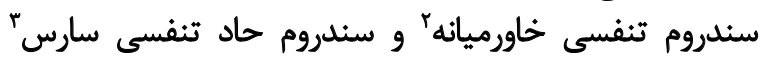

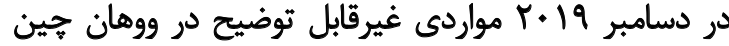

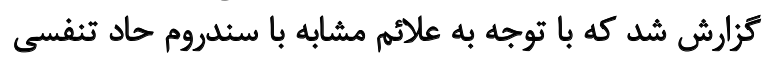

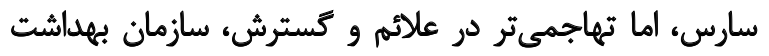

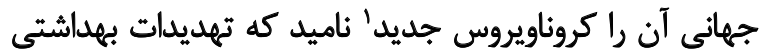

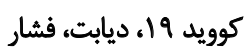

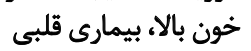

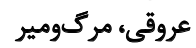




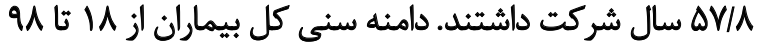

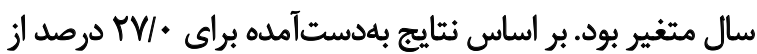

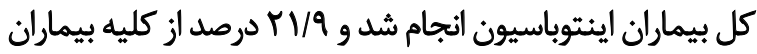

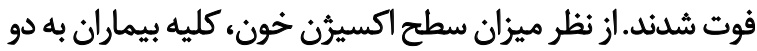

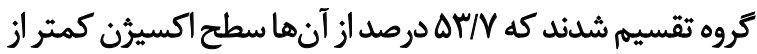

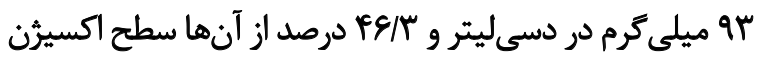

بيشتر از كاو ميلى گرم بر دسى ليتر و ليتر داشتند (جدول شماز انهاره ()). نتايج حاصل از بررسى تظاهرات بالينى بيماران نشان داد

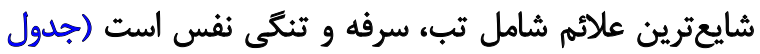

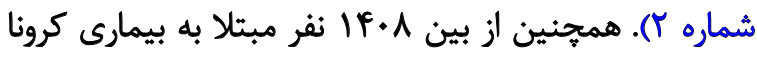

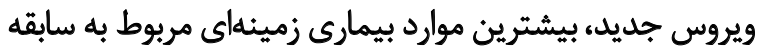

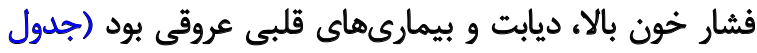

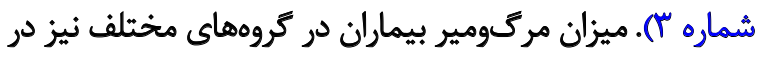
جدول شماره Ff آورده شده است.

بحث

كسترش جالشبرانكيز و جهانى كروناويروس باعث شده

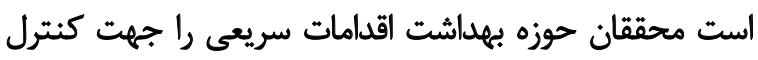

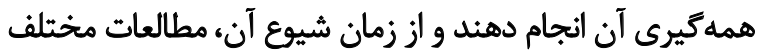

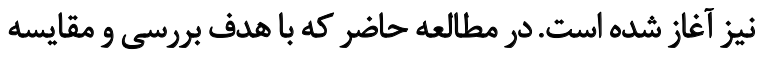

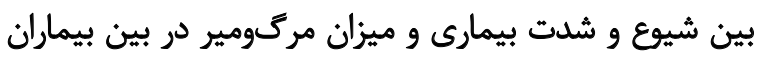

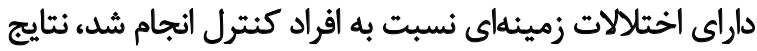

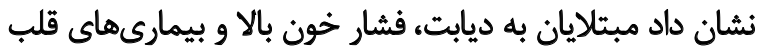

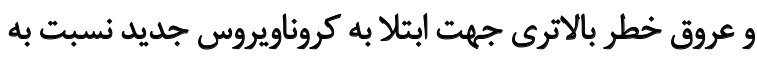

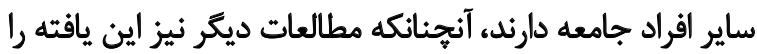

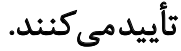

فشار خون بالا معمولاً با ديخر عوامل خطرساز همجئون

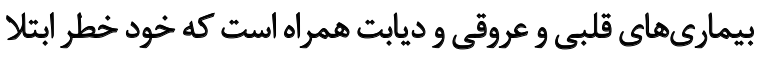

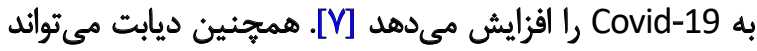

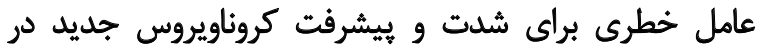

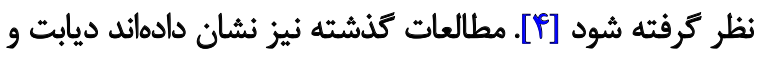

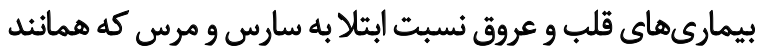

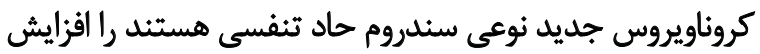

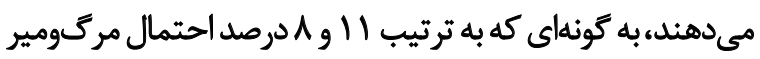

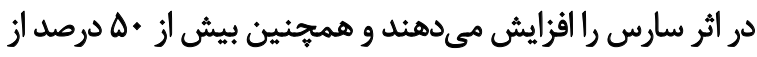

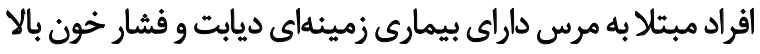

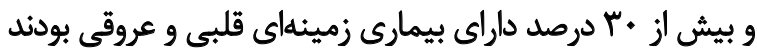

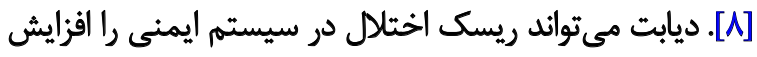

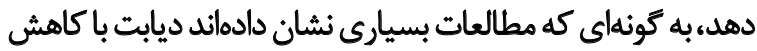

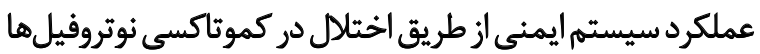

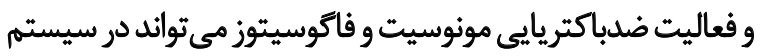

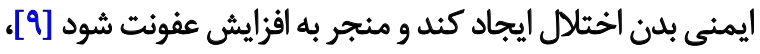

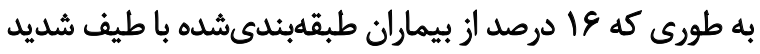

هستند. طبق آخرين آمار جهاني، كروناويروس جديد تا به امروز

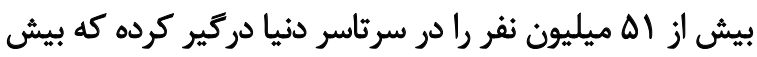

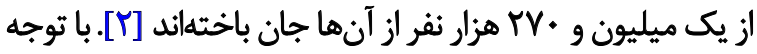

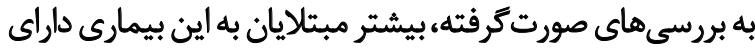

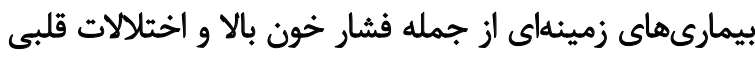

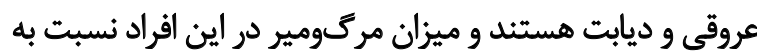

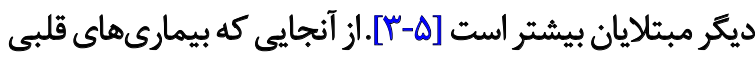

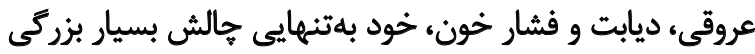

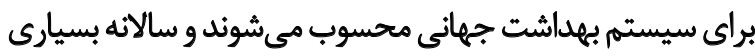

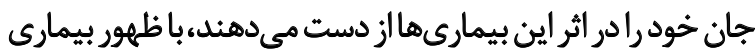

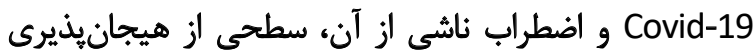

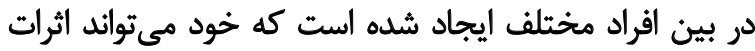

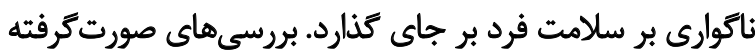

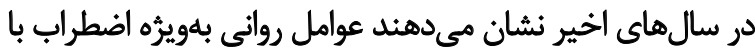

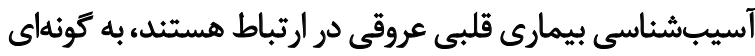

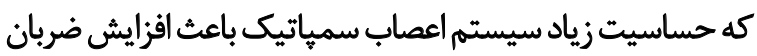

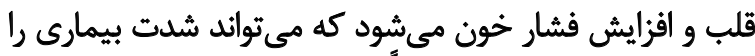

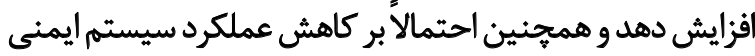

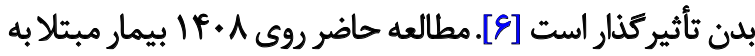

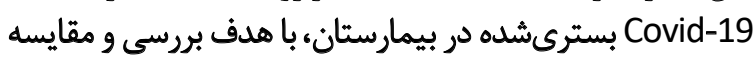

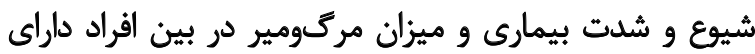
بيمارى زمينهاى نسبت به ديكر افراد مبتلا انجام شدر دئ

\section{مواد و روشُها}

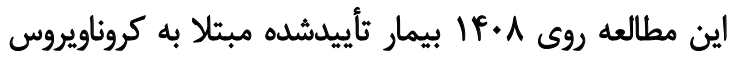

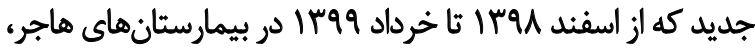

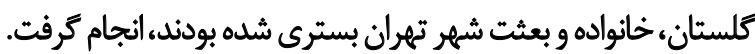

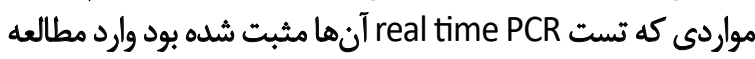

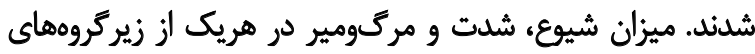

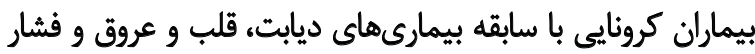

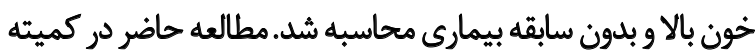

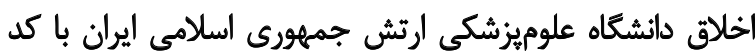

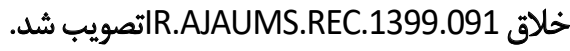
زادهها توسط آزمونهاى آمارى توصيفى و آزمونهاى تى

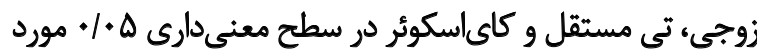

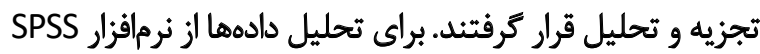
نسخخه وr استفاده شد.

\section{ياقتهها}

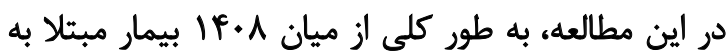

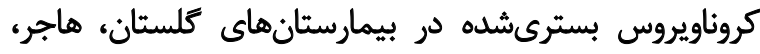

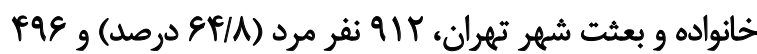

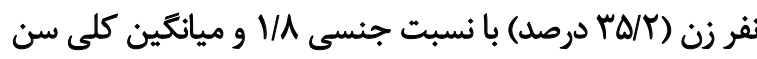


جدول ا. توزيع فراوانى متغيرهاي جمعيتشناختى كل بيماران مورد مطالعه

\begin{tabular}{|c|c|c|}
\hline تعداد (درصد) & \multicolumn{2}{|c|}{ متغير هاى جمعيتشناختى } \\
\hline $91 \%(g \times / A)$ & مرد & \multirow{2}{*}{ جنسيت } \\
\hline rag $(r o / T)$ & زن & \\
\hline $18(1 / 1)$ & كمتر از +r r & \multirow{6}{*}{ سن (سال) } \\
\hline $181(11 / 9)$ & ra-ri & \\
\hline MTP $(T M / \cdot)$ & Q.rE & \\
\hline $\operatorname{ref}(r \Delta / q)$ & $8 \Delta-\Delta 1$ & \\
\hline$m(r e / R)$ & A.-88 & \\
\hline $\operatorname{lef}(11 / 9)$ & بالاتر از • بال & \\
\hline $\operatorname{DAT}(\varepsilon \cdot / A)$ & بله & \multirow{2}{*}{ مصرف دخائيات } \\
\hline $1 \Delta P(M q / T)$ & خير & \\
\hline$r A \cdot(r V / \cdot)$ & بله & \multirow{2}{*}{ ايتتوباسيون } \\
\hline $1 . Y \wedge(M / 1)$. & خيخ & \\
\hline $11 \cdots(V N)$ & 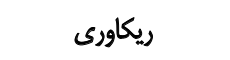 & \multirow{2}{*}{ وضعيت نهليع بيمار } \\
\hline$r+\Lambda(r / / q)$ & فوت & \\
\hline$V \Delta \&(\Delta M / V)$ & كمتر ازي سهو & \multirow{2}{*}{ 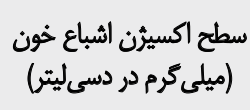 } \\
\hline $\operatorname{gar}\left(\operatorname{Fg} / \mathbb{T}^{\prime}\right)$ & مساوى يا بيشتر از تو & \\
\hline
\end{tabular}

جدول r. توزيع فراوانى علاثم مشاهدهشه در كل بيماران بسترى

\begin{tabular}{|c|c|c|}
\hline تعد/د (درصد) & & \\
\hline $\operatorname{Rr}($ SNT) & بله & \multirow{2}{*}{ تب } \\
\hline $\operatorname{Pro}\left(N^{\prime} / \mathcal{C}\right)$ & خير & \\
\hline $1 . \Delta A(V \Delta /))$ & بله & \multirow{2}{*}{ سرفه } \\
\hline$r \Delta \cdot(\pi F / q)$ & خير & \\
\hline $1 .+9(\mathrm{n} / \mathrm{N})$ & بله & \multirow{2}{*}{ تنكي نقس } \\
\hline req (Te/T) & خير & \\
\hline$m(19 / \%)$ & بله & \multirow{2}{*}{ سردرد } \\
\hline $11 \% \Delta(\Lambda \cdot / 8)$ & خير & \\
\hline$m(1 Q / T)$ & بله & \multirow{2}{*}{ حالت تههوع } \\
\hline $\operatorname{IIr}(A \cdot / \Lambda)$ & خير & \\
\hline $\mathrm{VA \Delta}(\Delta \Delta / A)$ & بله & \multirow{2}{*}{ ضعف و بيى حالى } \\
\hline err $(F \in \mathbb{R})$ & خير & \\
\hline $\operatorname{Mr}(I r / 9)$ & بله & \multirow{2}{*}{ درد قفسه سينه } \\
\hline $\operatorname{IrTE}(A V / I)$ & خير & \\
\hline$r+q(1 F / A)$ & بله & \multirow{2}{*}{ اسهال } \\
\hline $1199(1 \Delta / \pi)$ & خير & \\
\hline
\end{tabular}


جدول r. توزيع فراوانى اختلالات زمينهاي در بين بيماران

\begin{tabular}{|c|c|}
\hline تعداد (درصد) & ييمارى زمينهاى \\
\hline $\operatorname{meq}(r+/)$ & فشار خون بالا \\
\hline rq) $(r . / 1)$ & بيمارى هاى قليى عروقى \\
\hline$r . \&(T M N)$ & ديابت \\
\hline$\eta^{\sim}(\Delta / \pi)$ & سرطان \\
\hline$g r(F / A)$ & بيمارى كليوى \\
\hline
\end{tabular}

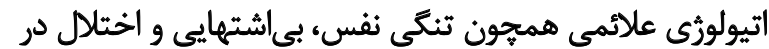

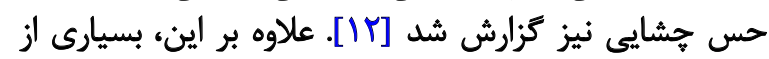

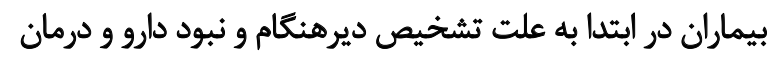

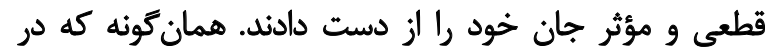

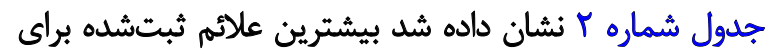

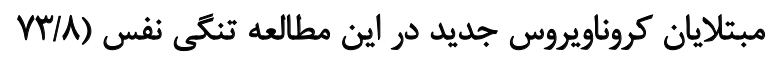

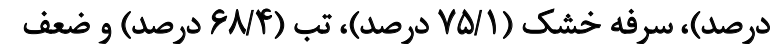

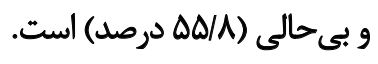

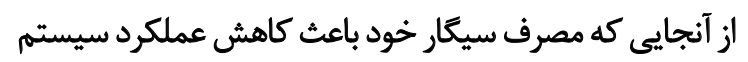

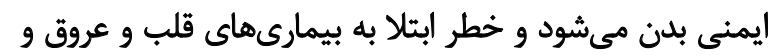

بيمارى كروناويروس در جين داراى بيمارى زمينهاى ديابت [ • [1]

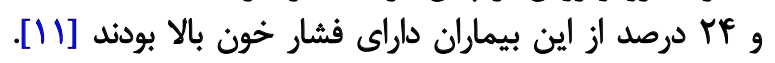

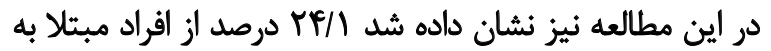

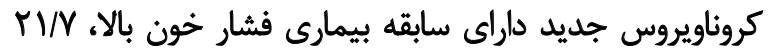

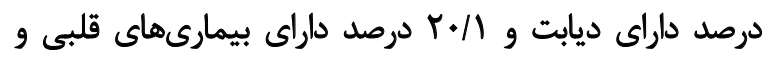

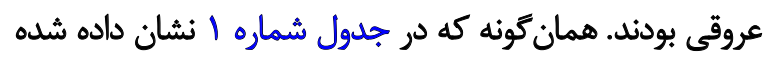

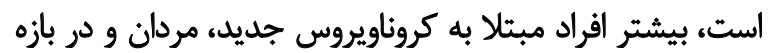
سنى •ه تا • م سال قرار دارند.

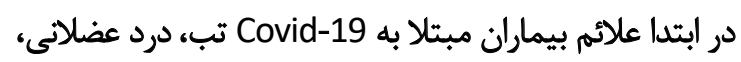

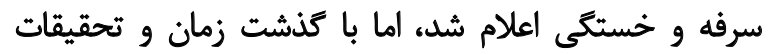

جدول F. توزيع فراوائى مركتومير در بين كروههاى مختلف

\begin{tabular}{|c|c|c|c|}
\hline سطح معنى فارى & تعداد (درصد) & \multicolumn{2}{|c|}{ متغيرها } \\
\hline . / apre & $\begin{array}{l}r+f(\& \& / M) \\
1 \cdot f(M T / A)\end{array}$ & مرد & جنسيت \\
\hline $.1 . \Delta<$ & 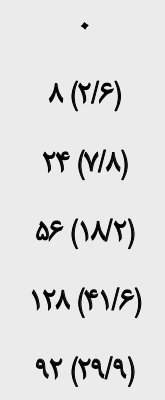 & 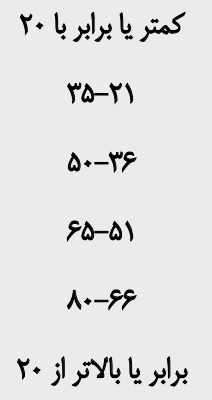 & سن (سال) \\
\hline $\begin{array}{l}.1 \cdot \Delta< \\
.1 \cdot \Delta< \\
.1 \cdot \Delta<\end{array}$ & $\begin{array}{l}A I \text { (TVIA) } \\
\text { GY (TNV) } \\
\text { AY (TEMA) }\end{array}$ & 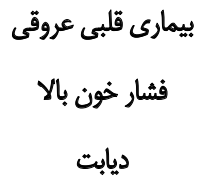 & اختلال زميئلهاى \\
\hline $.1 .0<$ & Im (re/l) & مصرف دخانيات & \\
\hline
\end{tabular}




$$
\text { (i) }
$$

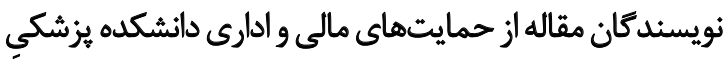

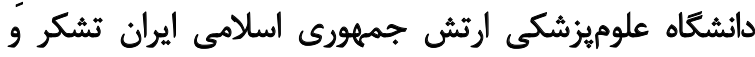
قدردانى مي كنئد.

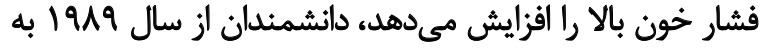

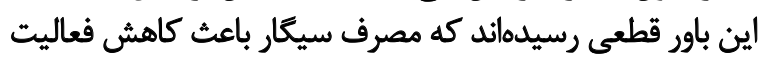

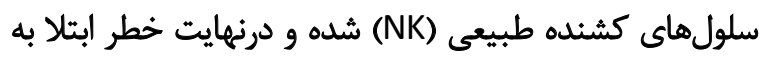

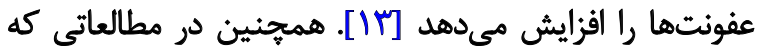

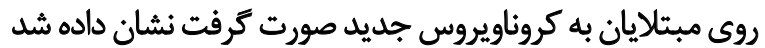

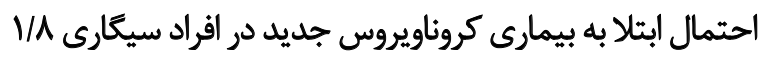
برابر بيشتر از ساير افراد است [If [ ]

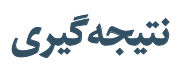

با توجه به نتايج بهدستآمده، وجود بيمارى هاى زيلى زمينهاى از

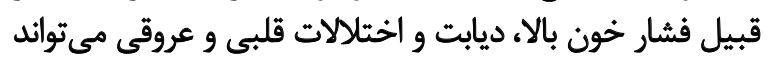

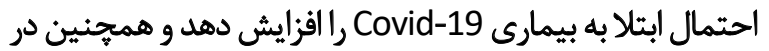

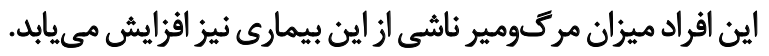

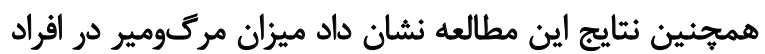
مصرفكنئده سيكار از افراد كنترل بيشتر است. بنابراين بيشنئهاد

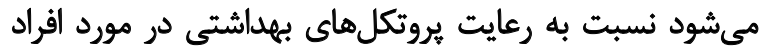

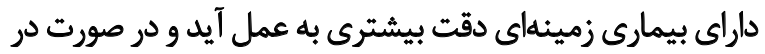

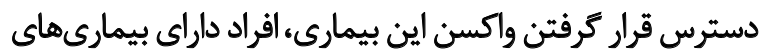
زمينهاى ذكرشده در اولويت قرار تيرند.

ماحظات اخلاقي

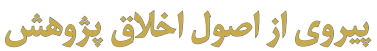

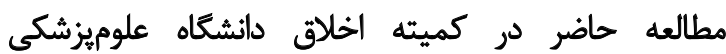

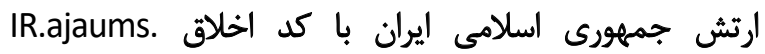

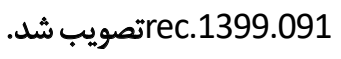

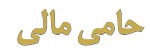

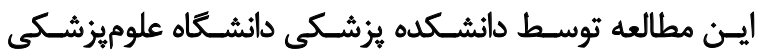

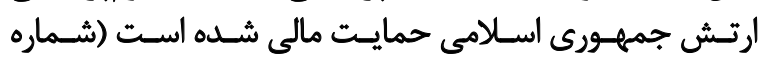

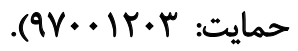

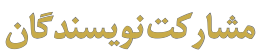

مفهوم سازى، روششناسى و تجزيه و تحليل دادهها: عمران

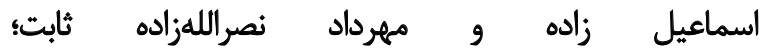

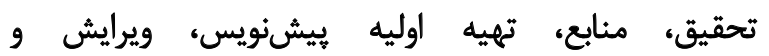

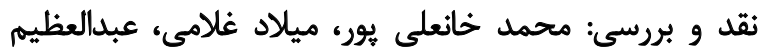
سرلى، عارفه رحيمى خرمى.

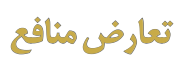
بنابر اظهار نويسندكان اين مقاله تعارض منافع ندارد. 


\section{References}

[1] Zhou P, Yang XL, Wang XG, Hu B, Zhang L, Zhang W, et al. A pneumonia outbreak associated with a new coronavirus of probable bat origin. $\mathrm{Na}$ ture. 2020; 579(7798):270-3. [DOI:10.1038/s41586-020-2012-7]

[2] WHO. Rolling updates on Coronavirus disease (COVID-19). [Internet]. 2020 [Updated 2020 July 31]. Available from: https://www.who.int/ emergencies/diseases/novel-coronavirus-2019/events-as-theyhappen

[3] South AM, Diz DI, Chappell MC. Chappell, COVID-19, ACE2, and the cardiovascular consequences. Am J Physiol Heart Circ Physiol. 2020; 318(5):H1084-90. [DOI:10.1152/ajpheart.00217.2020]

[4] Papadokostaki E, Tentolouris N, Liberopoulos E. COVID-19 and diabetes:What does the clinician need to know? Prim Care Diabetes. 2020; 14(5):558-63. [DOI:10.1016/j.pcd.2020.06.010]

[5] Clerkin KJ, Fried JA, Raikhelkar J, Sayer G, Griffin JM, Masoumi A, et al. COVID-19 and cardiovascular disease. Circulation. 2020. 141(20):164855. [DOI:10.1161/CIRCULATIONAHA.120.046941]

[6] Pfefferbaum B, North CS. Mental health and the Covid-19 pandemic. N Engl J Med. 2020; 383(6):510-2. [DOI:10.1056/NEJMp2008017]

[7] Ran J, Song Y, Zhuang Z, Han L, Zhao S, Cao P, et al. Blood pressure control and adverse outcomes of COVID-19 infection in patients with concomitant hypertension in Wuhan, China. Hypertens Res. 2020; 43(11):1267-76. [DOI:10.1038/s41440-020-00541-w] [PMCID]

[8] Park JE, Jung S, Kim A. MERS transmission and risk factors:A systematic review. BMC Public Health. 2018; 18(1):574. [DOI:10.1186/s12889018-5484-8]

[9] Pickup JC, Crook MA. Is type II diabetes mellitus a disease of the innate immune system? Diabetologia. 1998; 41(10):1241-8. [DOI:10.1007/ s001250051058]

[10] Guo W, Li M, Dong Y, Zhou H, Zhang Z, Tian C, et al., Diabetes is a risk factor for the progression and prognosis of COVID-19. Diabetes Metab Res Rev. 2020; e3319. [DOI:10.1002/dmrr.3319]

[11] Schiffrin EL, Flack JM, Ito S, Muntner P, Webb RC. Hypertension and COVID-19. Am J Hypertens. 2020; 33(5):373-4. [DOI:10.1093/ajh/ hpaa057]

[12] Hopkins C, Surda P, Kumar N. Presentation of new onset anosmia during the COVID-19 pandemic. Rhinology. 2020; 58(3):295-8. [DOI:10.4193/Rhin20.116]

[13] Tollerud DJ, Clark JW, Brown LM, Neuland CY, Mann DL, Pankiw-Trost LK, et al., Association of cigarette smoking with decreased numbers of circulating natural killer cells. Am Rev Respir Dis. 1989; 139(1):194-8. [DOI:10.1164/ajrccm/139.1.194]

[14] Vardavas Cl, Nikitara K. COVID-19 and smoking:A systematic review of the evidence. Tob Induc Dis. 2020; 18:20. [DOI:10.18332/tid/119324] 
This Page Intentionally Left Blank 